$63^{\text {ème }}$ Congrès de la SFCO, 02005 (2015)

DOI:10.1051/sfco/20156302005

(C) Owned by the authors, published by EDP Sciences, 2015

\title{
COMMUNICATION
}

\section{Intérêt de la décompression en orthodontie : à propos d'un cas de kératokyste chez l'enfant}

\author{
Alpy A, Pesudo M, L’Homme A, Vaysse F, Courtois B \\ Faculté dentaire, 3 chemin des maraichers, 31000 Toulouse
}

Les kératokystes représentent 4 à $10 \%$ de tous les kystes odontogéniques et se développent de manière asymptomatique dans la plupart des cas. Ils proviennent des résidus de la lame dentaire et de l'assise basale de l'épithélium buccal. Ils se caractérisent par rapport aux autres kystes odontogéniques par un fort taux de récidive. Différentes procédures chirurgicales sont décrites dans la littérature. L'énucléation est le traitement de choix d'exérèse du kératokyste. D’autres thérapeutiques sont proposées en fonction de différents paramètres comme la taille de la lésion, sa localisation, la proximité de structures nobles et l'âge du patient.

Les auteurs présentent une enfant de 9 ans qui consulte pour une gêne liée à la présence d'une fistule en regard de l'apex de la 34. Son chirurgien-dentiste traitant l'a traitée pour un abcès parodontal récidivant. Malgré un curetage parodontal, les récidives infectieuses se multiplient et la patiente est adressée à Toulouse pour une prise en charge hospitalière.

L'examen clinique montre l'existence d'une volumineuse tuméfaction mandibulaire asymptomatique du fond de vestibule qui s'étend de la 46 à la 34. L'orthopantomogramme montre une lésion radioclaire de $5 \mathrm{~cm}$ dans le sens mésiodistal, homogène, bien limitée, qui semble se développer à partir du sac péricoronaire de la 44 . Les dents 43,44 et 45 en position basale sont également incluses dans la lésion. Le CBCT confirme l'expansion en vestibulaire avec un respect de la corticale. Le diagnostic de kératokyste est posé cliniquement, radiologiquement puis confirmé par l'analyse anatomopathologique.

Dans ce contexte de lésion volumineuse, d'inclusion lésionnelle de trois dents définitives et prenant en compte l'âge de la patiente, la décompression s'impose comme la procédure chirurgicale la plus conservatrice au niveau osseux et dentaire. La mise en place d'un drain de décompression en polyéthylène au niveau du vestibule secteur 4 permet la réduction rapide du volume kystique. A la fois, les traitements chirurgical et orthodontique multibague bimaxillaire permettent l'éruption des dents définitives initialement concernées par le kératokyste. La coordination chirurgico-orthodontique a permis la disparition totale de la lésion kératokystique ainsi que la conservation et la mise en place des dents sur l'arcade. Deux ans après la décompression, une lacune essentielle de $2 \mathrm{~cm}$ dans le sens mésiodistal témoigne la rapidité de la réduction kystique qui a nécessité une intervention chirurgicale. La décompression, peu représentée dans la littérature, est une alternative conservatrice qui propose un certain nombre d'avantages: diminution du volume lésionnel, du risque fracturaire, du taux de récurrence par rapport à l'énucléation et la résection. Cette procédure favorise la poursuite de l'éruption dentaire ainsi que la modification de la nature histologique de la membrane kystique.

This is an Open Access article distributed under the terms of the Creative Commons Attribution License 4.0, which permits unrestricted use, distribution, and reproduction in any medium, provided the original work is properly cited. 
Cette technique nécessite la coopération totale du patient compte tenu de la durée importante du traitement et du suivi post opératoire. Parfois, la décompression s'associe à un geste thérapeutique secondaire. Bien que peu pratiquée de récentes études confirment l’efficacité du traitement à long terme.

Bibliographie

Gao \& al (2014)

Hou and Hongzhi Zhou (2013)

Kaczmarzyk \& al (2012)

Nakamura \& al (2002)

Nom et adresse du conférencier

Alpy ALEXANDRE

Faculté dentaire

3 chemin des maraichers

31000 Toulouse (France)

alexandre.alpy@yahoo.fr 